\title{
Dimensionality Reduction via the Laplace-Beltrami Operator: Application to EEG-based BCI
}

\author{
Xiaoqi Xu, Nicolas Drougard and Raphaëlle N. Roy
}

\begin{abstract}
Neural oscillations captured by electroencephalography (EEG) can be used by Brain-Computer Interfaces (BCIs) to reveal the underlying mental processes and enable explicitly or implicitly interacting with one's environment. Most feature extraction techniques are based on spatial filters and power analyses in multiple frequency bands. The global geometry feature is seldom investigated. In this paper, the spatial aspects of EEG signals are studied using the Laplace-Beltrami operator. The eigenvectors of the Laplace-Beltrami operator form an orthonormal basis for square-integrable functions over the scalp and capture the geometry of electrodes' position in a hierarchical way. The signals are decomposed into different spatial frequency components by the projection into the eigenspaces of the Laplace-Beltrami operator. Dimensionality reduction could be done by using only the low frequency components. This method is compared with Principal Component Analysis (PCA) filtering on publicly available motor imagery BCI data and achieved comparable results while being unsupervised, dataindependent and requiring $33.7 \%$ less computation time.
\end{abstract}

\section{INTRODUCTION}

Electroencephalography (EEG) records the electrical brain activity through electrodes placed along the scalp. Using features extracted from EEG signals, a Brain-Computer Interface (BCI) [1] can decode brainwaves into useful information to interact with one's environment. In BCI, as well as neuroscience, brainwaves are often analysed using timefrequency analysis. Different frequency bands, such as the well-known alpha and gamma waves are linked to different cognitive processes or states [2], [3]. On the contrary, the spatial frequency of EEG signals is rarely investigated in the literature. One of the reasons could be that the volume conduction makes the spatial interpretation of EEG signals more challenging [4]. Yet the spatial information could help better characterize relevant mental states for both clinical and everyday life applications, as well as increase the efficiency and robustness of active and passive BCIs.

The Laplace-Beltrami operator, a generalization of the Laplacian in Euclidean spaces to Riemannian manifolds, finds its origin in differential geometry. It encodes plenty geometric information of the underlying manifold and is extensively used in the field of computer graphics and geometry processing [5], [6], [7], [8], [9]. The eigenfunctions of the Laplace-Beltrami operator form an orthonormal basis for square integrable functions on the manifold. Moreover, the basis is well adapted to the geometry and the topology

\footnotetext{
*This work was supported by the ANITI, Artificial and Natural Intelligence Toulouse Institute, Université de Toulouse, France.

Xiaoqi $\mathrm{Xu}$, Nicolas Drougard and Raphaëlle N. Roy are with ISAE-SUPAERO, Université de Toulouse, France. (e-mail: firstname.surname@isae-supaero.fr).
}

of the manifold [6] and the eigenfunctions corresponding to larger eigenvalues have higher spatial variation.

For the neuroscience community, the Laplace-Beltrami operator is known under the name of Surface Laplacian and is mainly used as a spatial filter to mitigate volume conduction effects [10], [11], [4]. Its computation is also not very precise as it is supposed that the electrodes are placed over a sphere [10]. The most relevant work for this study was done by Graichen et al. [12]: they constructed a mesh from the coordinates of EEG electrodes and decomposed the EEG signals into the eigenspaces of the Laplace-Beltrami operator of the mesh. In their following work different methods of discretization of Laplace-Beltrami operator are compared [13]. They also compared the capability of dimensionality reduction of their method with principal component analysis (PCA) and independent component analysis (ICA) in terms of recovering total energy of the original EEG signal. However, no experiments were performed to show benefits for BCI applications and the noise suppression effects were only tested on artificial data. In a similar spirit, Huang et al. analyzed functional brain networks using graph frequency analysis [14]. They discovered a strong relationship between graph spectral properties and the level of exposure to tasks performed by the participants. However, they did not investigate their method for EEG signals, and the graph Laplacian used depended only on topology. We however advocate for the use of the discrete Laplacian that would keep the geometric properties of the underlying manifold.

\section{METHODS}

In the following, raw EEG signals with $n$ channels and $t$ time points are noted as $X \in \mathbb{R}^{n \times t}$. The Laplace-Beltrami operator is denoted by $\Delta$ in the continuous setting, and its matrix representation by $L \in \mathbb{R}^{n \times n}$ in the discrete setting. The $i^{\text {th }}$ eigenvector of the Laplace-Beltrami operator is denoted by $\phi_{i}, i \in\{1, \ldots, n\}$.

\section{A. Laplace-Beltrami operator}

Let $\mathcal{M}$ be a compact Riemannian manifold and $f \in$ $L^{2}(\mathcal{M})$ a square integrable function over $\mathcal{M}$. The LaplaceBeltrami operator is defined as a linear operator taking functions to the divergence of its gradient vector field:

$$
\Delta f=\operatorname{div}(\operatorname{grad} f) .
$$

Intuitively, the Laplace-Beltrami operator applied on a function $f$ measures the average extent to which the value of $f$ at a certain point deviates from the value of $f$ at nearby points. Since most natural phenomena, e.g. heat diffusion, 
have the property of depending on the difference of neighborhood points, this operator is ubiquitously used across many fields. However, in this work this operator will not be directly used as a filter. Instead, we use its eigenvectors, which are the analogue of trigonometric functions or spherical harmonics, to study the spatial properties of EEG signals and reduce their dimension as we detail in the next subsection.

By solving the eigenvalue problem

$$
\Delta f=\lambda f
$$

we get a series of non-negative and discrete eigenvalues $\lambda_{0}=0<\lambda_{1}<\ldots<\lambda_{i}<\ldots$, as well as the corresponding eigenvectors $\left\{\phi_{i}\right\}_{i \geqslant 0}$ which form an orthonormal basis for square integrable functions on the manifold. For any function $f \in L^{2}(\mathcal{M})$, we can rewrite it as $f=\sum_{i>0} c_{i} \phi_{i}$, where $c_{i}$ are the coefficients defined as the surface integral $c_{i}=\int_{\mathcal{M}} f \phi_{i}^{*}$, which describe the amplitude of $f$ in each component.

\section{B. Computation in the discrete setting}

The real scalp can be approximated by a triangle mesh constructed from the $3 \mathrm{D}$ coordinates of the electrodes in the standard 10-20 system. Since the Laplace-Beltrami operator is an important tool for the geometry processing community, its computation in the discrete setting is extensively studied [15], [16], [17]. Here we use the well-known cotangent formula [18], detailed in [5], for its good theoretical (convergence) properties and fast computation time.

The discrete Laplace-Beltrami operator is represented by a $n \times n$ matrix $L$ where $n$ is the number of nodes (or vertices), representing electrodes in our case. It is defined by $L=$ $S^{-1} M$ where $M$ is the cotangent weights matrix and $S$ the area matrix. The cotangent weights matrix $M$ is defined by

$$
M_{i j}= \begin{cases}\sum_{k \neq i} m_{i k} & \text { if } i=j \\ -m_{i j} & \text { if } i \text { and } j \text { adjacent } \\ 0 & \text { otherwise. }\end{cases}
$$

where $m_{i j}$ are cotangent weights: $m_{i j}=\frac{1}{2}\left(\cot \left(\alpha_{i j}\right)+\right.$ $\left.\cot \left(\beta_{i j}\right)\right)$ when $i$ and $j$ are adjacent and $m_{i j}=0$ otherwise. The area matrix $S$ is the diagonal matrix with diagonal values $s_{i}$, which is sum of area of small patches surrounding the $i^{t h}$ vertex (see [18] for more details).

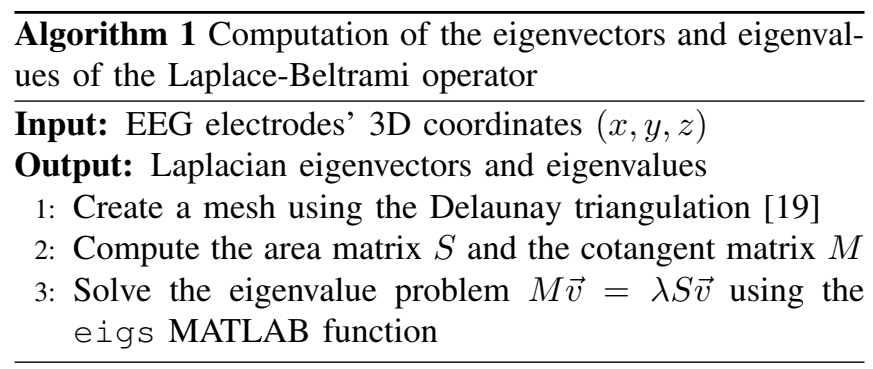

The discrete version of the eigenvalue problem (Equation 1) is $L \vec{v}=\lambda \vec{v}$ and can then be rewritten as a generalized eigenvalue problem $M \vec{v}=\lambda S \vec{v}$. This can be solved efficiently by the Arnoldi method of ARPACK (implemented
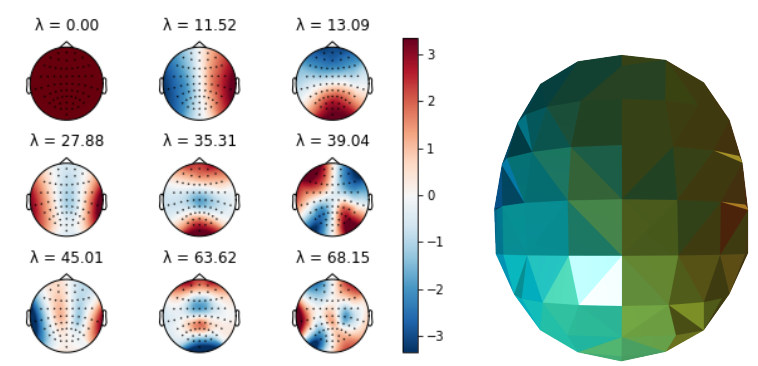

(a) Topomaps of the first 9 Laplacian (b) Triangle mesh coneigenvectors and eigenvalues sidered (top view)

Fig. 1: (a) Topomaps of the first 9 eigenvectors and eigenvalues of the Laplace-Beltrami operator. (b) Underlying mesh constructed using the MI dataset montage [20], [21]. Note: first eigenvector always constant and the spatial variation of the eigenvector increases with the corresponding eigenvalue.

inside MATLAB). In the following, the discrete LaplaceBeltrami operator is called Laplacian for short. In Fig. 1, we show the topomaps of the first 9 eigenvectors and corresponding eigenvalues of the Laplacian computed as described in Algorithm 1. The first eigenvector that corresponds to the eigenvalue 0 is always a constant vector. The larger the eigenvalue is, the larger spatial variation the eigenvector has.

\section{Application to EEG signals}

Traditionally, EEG signals $X \in \mathbb{R}^{n \times t}$ are regarded as a set of time series data. This point of view neglects the geometric information of the electrodes' positions. If EEG signals are thought of as functions over the manifold of the scalp, then their coefficients, or projection, in each Laplacian eigenspace constitute a decomposition of the signals into different spatial frequency components. The matrix of Laplacian eigenvectors is denoted by $\Phi=\left[\phi_{1}, \ldots, \phi_{n}\right] \in \mathbb{R}^{n \times n}$. Then, the coefficient matrix:

$$
C=\Phi^{\top} X
$$

is the projection of the original signal into the Laplacian spectral domain. Hence, the original signal can be reconstructed without loss. Each row (coefficients in each eigenspace) represents the evolution over time of a certain spatial frequency component.

As we mentioned in the introductory section, time frequency bands (e.g. alpha band) have been studied extensively [22], [23], [24], but very little is known about the spatial properties of EEG signals. Using the Laplacian spectral coefficients well suited to analyse EEG signals spatially, we explored several aspects:

- The average power in the Laplacian spectral domain is computed. The spectral coefficients are squared to remove sign ambiguity and averaged over trials.

- Elementary experiments are done to test classical EEG signal classification methods in the spectral domain. Two golden standard classification methods for BCI are used as baseline: Common spatial patterns (CSP) with linear discriminant analysis (LDA) [25], and Minimum Distance to Mean with geodesic filtering (FgMDM) 
[26]. Even though spectral decomposition via Laplacian keeps essentially the same information of the original signal, using the above classification methods in the spectral domain has a different interpretation compared to using them in the classical spatial domain. CSP in the spectral domain could be seen as finding a linear combination of spectral components that best differentiate between classes. And the covariance matrix used in FgMDM contains the correlation coefficients of pairs of spectral components instead of pairs of spatial components (i.e. electrodes).

- Lastly, dimensionality reduction can be done by keeping only the first spatial frequency components, as most information is retained by the smoothest components (cf. section III-B and Algorithm 2). This can induce a low-pass effect on signals thus naturally suppressing noise and leading to better classification results.

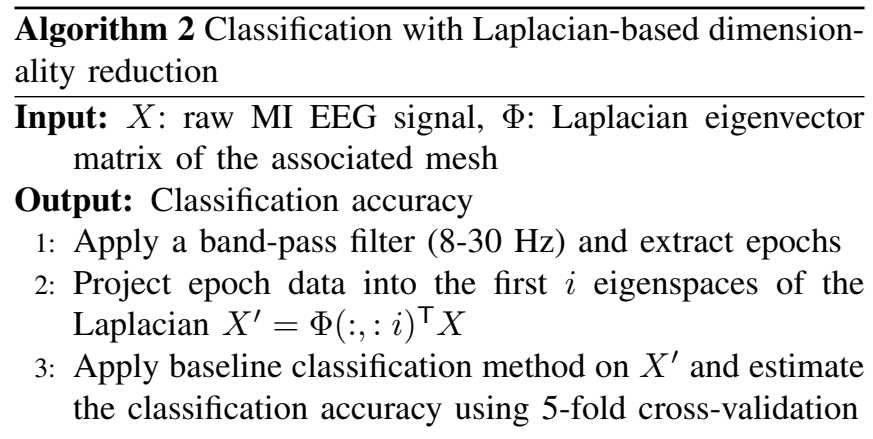

\section{EXPERIMENTS AND RESULTS}

\section{A. Data and preprocessing}

To promote the reproducibility of results and facilitate comparison with other methods, our experiments were based on a publicly available dataset: Physionet EEG motor imagery dataset [20], [21]. It contains over 1500 one- and 2minute EEG recordings, obtained from 109 volunteers. 64channel data were recorded using the BCI2000 system while subjects performed different MI tasks. In our experiments, we used only trials concerning the MI of opening and closing either both fists or both feet. No specific prepossessing was used for all experiments. Raw data was read and split into epochs according to event annotations. A band-pass filter was used to extract the $\alpha$ and $\beta$ bands $(8-30 \mathrm{~Hz})$ since they are particular discriminant for motor imagery [27].

\section{B. Spatial analysis}

For the MI EEG signals, the average power in the spectral domain presented strong common patterns across individuals. Fig. 2 (b) shows the mean (over subjects) average (over trials) power of the MI EEG signals in the spectral domain. Two high peaks can be noticed over the $3^{\text {rd }}$ and $5^{\text {th }}$ Laplacian eigenspaces. The topomaps of these eigenvectors are shown at the top row in the middle of the same figure. $\phi_{3}$ captures features along the longitudinal axis, while $\phi_{5}$ captures differences along the lateral axis (near the center of the brain). This pattern could reflect the greater involvement of the motor areas - located at central sites - during motor imagery compared to other areas. This would explain also the greater spectral power for the $5^{\text {th }}$ eigenspace in the hands MI condition as compared to the feet one (as seen in Fig. 2 (b). Indeed, given that the feet area is located deeper into the interhemispheric fissure, feet MI mostly impacts the vertex electrode while hands MI impacts the lateral ones (i.e. C3 and C4). There are also some small peaks over the high frequency eigenvectors whose topomaps are shown at the bottom of Fig. 2 (a). These eigenvectors highlight the C6 and FC6 electrode sites, i.e. electrodes on the right hemisphere which might also play an important role during MI tasks, irrespective of the imagined body part.

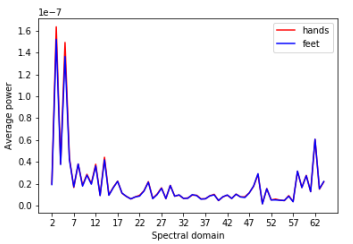

(a) Avg power per spectral domain

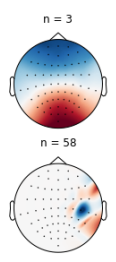

(b) Eigenvectors $\phi_{3}, \phi_{5}, \phi_{58}, \phi_{62}$
Fig. 2: Average power of MI EEG signals in the spectral domain and their corresponding eigenvectors. Power averaged over 45 trials of hands/feet MI over 109 subjects [20], [21]. In (a) the values for $n=1$ are omitted to allow correct visualization (hands: $9.003 \times 10^{-7}$; feet: $8.604 \times 10^{-7}$ ).

\section{Classification and dimensionality reduction}

The binary (i.e. hands vs feet) classification accuracy of baseline classification methods applied on the Motor Imagery EEG dataset and on an increasing number of PCA or Laplacian spectral components is shown in Fig. 3. This was estimated using a 5-fold cross-validation procedure. Note that for CSP+LDA, applying dimensionality reduction before the classifier did not lead to a significant increase in the classification accuracy. This could find an explanation in [25] where the authors explain that in theory any prefiltering either harms or does not change the performance of the CSP. Indeed, applying a prefiltering or projection $P$, whether invertible or not, we always have:

$$
\max _{\tilde{w}} \frac{\tilde{w}^{\top} P^{\top} \Sigma_{1} P \tilde{w}}{\tilde{w}^{\top} P^{\top} \Sigma_{2} P \tilde{w}} \leq \max _{w} \frac{w^{\top} \Sigma_{1} w}{w^{\top} \Sigma_{2} w} .
$$

The improvement of classification accuracy might result from noise reduction as a byproduct of using the smoothest spatial frequency components. On the contrary, for FgMDM, classification accuracy was substantially increased after applying dimensionality reduction. This indicates that the covariance matrix of smooth components contains much less noise. Moreover, the computation time can be considerably reduced, especially for FgMDM, see Fig. 3 (b) (computation done on a desktop computer with Inter Core i7$4790 \mathrm{CPU}$ and 15.6 GB of RAM memory). The best number of components to use can be chosen by cross validation. The classification pipeline with the proposed method achieves 


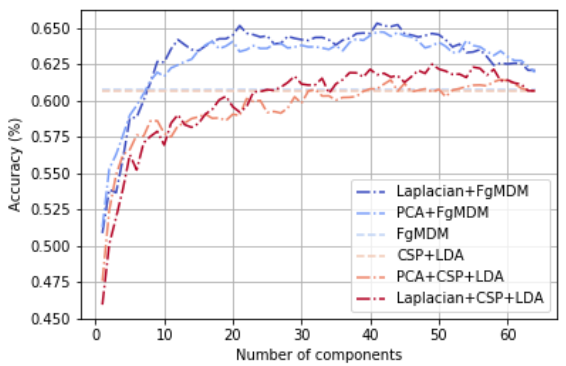

(a) Classification accuracy

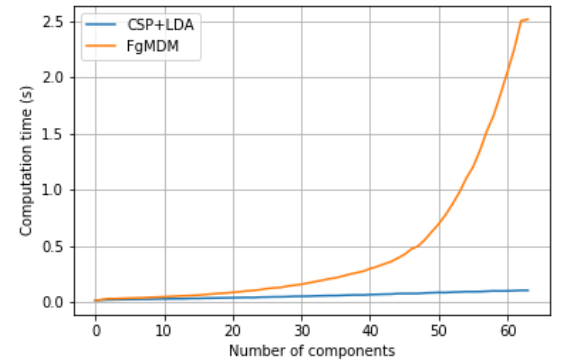

(b) Computation time

Fig. 3: (a) Classification accuracy as a function of the number of PCA or Laplacian spectral components used for dimensionality reduction, averaged over 109 subjects. Baseline: CSP+LDA and FgMDM respectively. (b) Computation time depending on the number of kept Laplacian spectral components.

comparable results compared to the one with PCA while requiring $33.7 \%$ less computation time.

\section{CONCLUSION AND FUTURE WORK}

In this paper, the possibility of using the Laplace-Beltrami operator as a geometric tool to study the spatial properties of EEG signals for MI-based BCI has been explored. Interestingly, the average power in the spectral domain coincides with the physiology knowledge of the underlying brain activity. Moreover, dimensionality reduction can be done by passing a low-pass filter in the Laplacian spectral domain. The results showed the efficiency of the proposed method and an improved classification accuracy in BCI applications. An interesting perspective would be to compare or integrate this type of operator with Geometric Deep Learning methods, which tends to generalize deep learning methods to nonEuclidean domains.

\section{REFERENCES}

[1] J. R. Wolpaw, N. Birbaumer, W. J. Heetderks, D. J. McFarland, P. H. Peckham, G. Schalk, [...], and T. M. Vaughan, "Brain-computer interface technology: a review of the first international meeting," IEEE Trans Rehab Eng, vol. 8, no. 2, pp. 164-173, 2000.

[2] E. Başar, C. Başar-Eroglu, S. Karakaş, and M. Schürmann, "Gamma, alpha, delta, and theta oscillations govern cognitive processes," Int $J$ Psychophysiol, vol. 39, no. 2, pp. 241 - 248, 2001.

[3] L. M. Ward, "Synchronous neural oscillations and cognitive processes," Trends Cogn Sci, vol. 7, no. 12, pp. 553 - 559, 2003.

[4] B. Burle, L. Spieser, C. Roger, L. Casini, T. Hasbroucq, and F. Vidal, "Spatial and temporal resolutions of eeg: Is it really black and white? a scalp current density view," Int J Psychophysiol, vol. 97, no. 3, pp. $210-220,2015$.

[5] R. M. Rustamov, "Laplace-Beltrami Eigenfunctions for Deformation Invariant Shape Representation," in Geometry Processing, A. Belyaev and M. Garland, Eds. The Eurographics Association, 2007.

[6] B. Levy, "Laplace-beltrami eigenfunctions towards an algorithm that "understands" geometry," in IEEE Int Conf Shape Modeling and Applications, 2006, pp. 13-13.

[7] M. Reuter, S. Biasotti, D. Giorgi, G. Patanè, and M. Spagnuolo, "Discrete laplace-beltrami operators for shape analysis and segmentation," Computers \& Graphics, vol. 33, no. 3, pp. 381 - 390, 2009.

[8] M. Reuter, F.-E. Wolter, and N. Peinecke, "Laplace-beltrami spectra as 'shape-dna' of surfaces and solids," Computer-Aided Design, vol. 38, no. 4, pp. $342-366,2006$.

[9] M. Ovsjanikov, M. Ben-Chen, J. Solomon, A. Butscher, and L. Guibas, "Functional maps: A flexible representation of maps between shapes," ACM Trans. Graph., vol. 31, no. 4, 2012.
[10] C. Carvalhaes and J. A. de Barros, "The surface laplacian technique in eeg: Theory and methods," International Journal of Psychophysiology, vol. 97 , no. 3, pp. 174-188, 2015.

[11] M. X. Cohen, Analyzing Neural Time Series Data. MIT Press, 2014.

[12] U. Graichen, R. Eichardt, P. Fiedler, D. Strohmeier, and J. Haueisen, "Adaptive spatial harmonic analysis of eeg data using laplacian eigenspace," in Int Symp Noninvasive Functional Source Imaging Brain Heart, 2011, pp. 18-21.

[13] U. Graichen, R. Eichardt, P. Fiedler, D. Strohmeier, F. Zanow, and J. Haueisen, "Sphara-a generalized spatial fourier analysis for multisensor systems with non-uniformly arranged sensors: application to eeg," PloS one, vol. 10, no. 4, p. e0121741, 2015.

[14] W. Huang, L. Goldsberry, N. F. Wymbs, S. T. Grafton, D. S. Bassett, and A. Ribeiro, "Graph frequency analysis of brain signals," IEEE $J$ Selected Topics Sign Proc, vol. 10, no. 7, p. 1189-1203, 102016.

[15] G. Xu, "Convergence of discrete laplace-beltrami operators over surfaces," Comput Math Appl, vol. 48, no. 3-4, pp. 347-360, 2004.

[16] M. Desbrun, M. Meyer, P. Schröder, and A. H. Barr, "Implicit fairing of irregular meshes using diffusion and curvature flow," in Proc Annual Conf Computer Graphics and Interactive Techniques. USA: ACM Press/Addison-Wesley Publishing Co., 1999, p. 317-324.

[17] M. Meyer, M. Desbrun, P. Schröder, and A. H. Barr, "Discrete differential-geometry operators for triangulated 2-manifolds," in $\mathrm{Vi}$ sualization and Mathematics III, H.-C. Hege and K. Polthier, Eds. Berlin, Heidelberg: Springer Berlin Heidelberg, 2003, pp. 35-57.

[18] G. Xu, "Discrete laplace-beltrami operator on sphere and optimal spherical triangulations," Int J Comput Geom \& Appl, vol. 16, no. 01, pp. 75-93, 2006.

[19] D. Lee and B. Schachter, "Two algorithms for constructing a delaunay triangulation," Int J Comput Information Sci, 1980.

[20] A. Goldberger, L. Amaral, L. Glass, J. Hausdorff, [...], and H. E. Stanley, "Physiobank, physiotoolkit, and physionet: Components of a new research resource for complex physiologic signals." 2000.

[21] G. Schalk, D. J. McFarland, T. Hinterberger, N. Birbaumer, and J. R. Wolpaw, "Bci2000: a general-purpose brain-computer interface (bci) system," IEEE Trans Biomed Eng, vol. 51, no. 6, pp. 1034-1043, 2004.

[22] W. H. R. Miltner, C. Braun, M. Arnold, H. Witte, and E. Taub, "Coherence of gamma-band EEG activity as a basis for associative learning," Nature, 1999.

[23] W. Klimesch, H. Schimke, and J. Schwaiger, "Episodic and semantic memory: an analysis in the eeg theta and alpha band," Electroencephalogr Clin Neurophysiol, vol. 91, no. 6, pp. 428 - 441, 1994.

[24] W. Klimesch, M. Doppelmayr, H. Russegger, T. Pachinger, and J. Schwaiger, "Induced alpha band power changes in the human eeg and attention," Neurosci Lett, vol. 244, no. 2, pp. 73 - 76, 1998.

[25] B. Blankertz, R. Tomioka, S. Lemm, M. Kawanabe, and K. Muller, "Optimizing spatial filters for robust eeg single-trial analysis," IEEE Signal Processing Magazine, vol. 25, no. 1, pp. 41-56, 2008.

[26] A. Barachant, S. Bonnet, M. Congedo, and C. Jutten, "Riemannian geometry applied to BCI classification," in Latent Variable Anal and Signal Sep. Springer Berlin Heidelberg, 2010, pp. 629-636.

[27] G. Pfurtscheller and C. Neuper, "Motor imagery and direct braincomputer communication," Proc IEEE, vol. 89, pp. 1123-1134, 2001. 